\begin{tabular}{|c|c|c|}
\hline & Int.J.Curr.Microbiol.App.Sci (2021) 10(08): xx-xx & \\
\hline & $\begin{array}{l}\text { International Journal of Current Microbiology and Applied Sciences } \\
\text { ISSN: 2319-7706 Volume } 10 \text { Number } 08 \text { (2021) } \\
\text { Journal homepage: http://www.ijcmas.com }\end{array}$ & 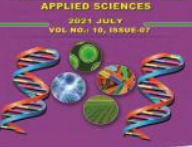 \\
\hline $\begin{array}{l}\text { EXCELLENT } \\
\text { PUBLISHERS }\end{array}$ & & wurw ijemas.com \\
\hline
\end{tabular}

Original Research Article https://doi.org/10.20546/ijcmas.2021.1008.025

\title{
Awareness, Knowledge and Attitude of Breast-Feeding Practices among Post Natal Mothers
}

\author{
Vandana Gupta*
}

Department of Home Science, Dev Samaj College for Women, Ferozepur City, Punjab, India

*Corresponding author

\section{Keywords}

Exclusive Breast

Feeding,

Knowledge,

Maternal Attitude

Article Info

Accepted:

15 July 2021

Available Online:

10 August 2021

\section{A B S T R A C T}

Breastfeeding is one of the most important determinants of child survival, birth spacing, and prevention of childhood infections. The importance of exclusive breastfeeding and the immunological and nutritional values of breast milk have been well demonstrated by various intense researches. The WHO recommends that for the first six months of life, infants should be exclusively breastfed to achieve optimal growth, development, and health. Six months onwards the infants should be given nutritionally adequate and safe supplementary foods, while continuing to breastfeed for up to two years or beyond. A number of studies have been assessed regarding knowledge, attitude and breastfeeding practices in different parts of the world; such studies are limited among Indian mothers. Especially the studies on breastfeeding awareness, knowledge and maternal attitude are also a concept of interest to health professionals who support breastfeeding. Therefore, this community based cross sectional study was undertaken to examine the infant feeding practices, knowledge and attitude towards breast feeding among Indian postnatal mothers residing in urban and well as rural areas of Ferozepur district.. On the basis of random sampling technique 300 respondents were selected from the Ferozepur district which was further divided into six different blocks (Guruharsahai, Zira, Makhu, Ghalkhurd, Mamdot and Ferozepur cantt). All 300 respondents were covered recently from all blocks of the Ferozepur district including approximately 50 nursing mothers from each block respectively. Data was collected by personally administering the questionnaire of nursing mothers of different areas of respective blocks. Higher educational qualification leads to increased mean awareness score pertaining to breast feeding of nursing respondents $(\mathrm{p}<0.01)$ The mean knowledge scores of nursing mothers regarding breast feeding does not depend on the increase in the level of education. ( $\mathrm{p}<0.01)$ There is pertinent need to spread awareness in their local language regarding specific terms like "exclusive breast feeding" and "infant formula feeding" among nursing mothers. 


\section{Introduction}

Breastfeeding is the natural feeding method and breast milk is the best milk for infants (Datta, 2009). According to WHO (2016) reported that breastfeeding is a unique, valuable feeding practice in infancy stage i.e. linked with lower neonatal mortality and which prevents morbidities and alleviates inequities in child mortality and such as neonatal sepsis diarrhea, pneumonia, and might reduce the chances of obesity and diabetes later in life. Initiation of breastfeeding, as early as possible has become mandatory because it serves multiple purposes to the growing baby, mother, and also the whole family as per baby-friendly initiative norms, Exclusive breastfeeding for the first six months of life followed by safe complementary foods and nutritionally adequate with continued breastfeeding up to 2 years of age or beyond is the recommended practice by American Academy of Paediatrics and World Health Organization.

Though Malnutrition among the children has reduced significantly in India over the years, but still the number of malnourished children is extremely high in the country. Mortality among infants and under-5 children is also a major concern. In India the number of under-5 mortality rate and infant mortality rates are 49 and 42 , respectively. A look at the above figures calls for giving attention to the breastfeeding practices in the population. An estimated $11.6 \%$ of infant deaths and 21.9 million disability-adjusted years could be prevented by large-scale breastfeeding promotion programmes (WHO 2009).

The risk of early death from diarrhea, respiratory diseases and other common childhood illnesses rises dramatically among children not breastfed as compared to children who are exclusively breastfed. Late initiation of breastfeeding not only deprives the child of valuable feed, but also leads to introduction of pre-lacteal feeds (that is, something other than breast milk) like glucose water, honey, ghutti, animal milk, or powdered milk that are potentially harmful and contribute to diarrhoea in the newborn. Breastfeeding significantly shields against neonatal sepsis, pneumonia, and diarrhoea which was emphasized in Lancet's "Child Survival Series" of 2003. Breast milk provides all essential nutrients for infants less than six months of age (World Health Organization, 2016).

Breastfeeding is one of the smartest investments that any country/ society can make to ensure a healthy, equitable and sustainable future. As is well documented that mother's milk provides a blanket protection to the child from various minor and major health and developmental problems and complications, but the information on the prevalence rate of breastfeeding and factors influencing the breastfeeding practices is limited, especially in the rural and far flung areas of India. A number of studies have examined the different breastfeeding practices in different part of world, but this typeof studies are limited among Indian postnatal mothers. Especially studies on breastfeeding knowledge, practices, attitude of mothers, maternal attitude are also concept of interest to health professionals who support breastfeeding. The objective of this minor research project is to assess the prevalence and examine various practices associated with exclusive breastfeeding during the first six months of life among mothers in urban and rural areas of Ferozepur City of Punjab- a backward and border city of India

To examine the knowledge and attitude towards breastfeeding amongst nursing mothers from different socio-economic backgrounds. To Compare the breastfeeding practices awareness, knowledge and attitude score across various categories 
Alison et al., (2019) conducted systematic search of six electronic databases, randomised controlled trials comparing breastfeeding counselling with no breastfeeding counselling or different formulations of counselling were between birth and 24 months after birth. There was a significant effect of counselling interventions on any breastfeeding at 4 to 6 weeks (Relative risk [RR] 0.85, 95\% CI 0.77, 0.94 ) and 6 months (RR 0.92, 95\% CI 0.87 , 0.94). Greater effects were found on exclusive breastfeeding at 4 to 6 weeks (RR $0.79,95 \%$ CI $0.72,0.87$ ) and 6 months (RR $0.84,95 \%$ CI $0.78,0.91)$. Counselling delivered at least four times postnatally is more effective than counselling delivered antenatally only and/or fewer than four times. Breastfeeding counselling is an effective public health intervention to increase rates of any and exclusive breastfeeding. Breastfeeding counselling should be provided face-to-face, and in addition, may be provided by telephone, both antenatally and postnatally, to all pregnant women and mothers with young children to inform scale-up globally there is a need to further understand the elements of breastfeeding interventions such as counselling and their effectiveness in different contexts and circumstances.

Joseph et al., (2019) studied that the sociocultural factors that influence exclusive breastfeeding among rural mothers. Suboptimal breastfeeding is responsible for $96 \%$ of deaths among children under 12 months of age in developing countries. The breastfeeding initiation - the determinants of how soon a mother initiated breastfeeding included traditional new-born care practices, the birth attendant and place of delivery, exclusive breastfeeding - motivation to sustain exclusive breastfeeding was influenced by the conflict between the obligation to perform traditional rites, the mother's awareness and family support and decision making about infant feeding - the husband, grandmother, traditional birth attendant and the health workers all influenced participants' decisions around infant feeding. The key role that grandmothers and husbands have in decisions about breastfeeding demonstrates the need to engage the support of partners and relatives through community-driven policies and integrated interventions that address social and cultural barriers throughout the prenatal and postnatal period.

Milinco et al., (2019) studied the prevalence of breastfeeding in a baby friendly pediatric practice. In a pediatric practice in Italy, actions were undertaken to apply the recommendations for a breastfeeding-friendly physician's office and to promote the adoption of a semi-reclined or laid-back maternal position in breastfeeding. Result showed that a total of 265 newborn infants with a gestational age greater than 30 weeks were registered with the pediatric practice, about $18 \%$ of all infants born in Trieste in that period. The rate of exclusive breastfeeding at five months of age was higher than the one reported for the whole infant population of Trieste and of the Friuli Venezia Giulia Region $(62.3 \%$ vs. $42.9 \%$ vs. $30.3 \%)$ in the same period. The implementation of breastfeeding-friendly pediatric practice and the application of laidback breastfeeding may improve the rate and duration of exclusive breastfeeding.

Craig, (2010) aimed to uncover the perceived usefulness of a contemporary antenatal education strategy for mother's experience of breastfeeding initiation. This was a simple descriptive pilot study with ten first time mothers as participants; all of who were booked into an Australian private maternity unit for antenatal breastfeeding education, labour, birth and postpartum care. Semistructured interviews were transcribed verbatim and thematically analyzed. The findings of the study were antenatal education was beneficial for informing first time mothers 
of the practical skills required to positively initiate breastfeeding.

According to WHO report India is among the world's five largest emerging economies where investment in breastfeeding is significantly low, resulting in an annual economic loss of $\$ 14$ billion because of child deaths and cognitive losses caused from poor breastfeeding practices.

On the Global Hunger Index (2016) India is on place 67 among the 80 nations having the worst hunger situation which is worse than nations such as North Korea or Sudan and in 2016 it has slipped down to 97 among 118 countries which is alarming. Since 1990 there have been some improvements for children, but the proportion of hungry in the population has increased. In India $44 \%$ of children under the age of 5 are underweight. $72 \%$ of infants and $52 \%$ of married women have anaemia. Research has conclusively shown that malnutrition during pregnancy causes the child to have increased risk of future diseases, physical retardation, and reduced cognitive abilities It is evident that inappropriate breastfeeding practices are associated with severe malnutrition in the under five children, lack any advantage in terms of weight gain and are associated with growth faltering

\section{Materials and Methods}

For achieving the objectives of the study a random selection of 300 nursing mothers was done from the Ferozepur district of Punjab which was further divided into six different blocks (Guruharsahai, Zira, Makhu, Ghalkhurd, Mamdot and Ferozepur cantt) including approximately 50 nursing mothers from each block respectively. The respondents were administered with a pre-structured nondisguised questionnaire validated through a pilot survey to fill out a brief survey about the maternal background information, mother's awareness, mother knowledge, attitude of nursing mothers, information regarding breast feeding in hospitals, home, public places, breast feeding practices among mothers, types of supplementary foods given to the child, prevalence of breastfeeding practices and child disease incidences.

For collecting data from the nursing mothers, the respondents were administered with a prestructured non-disguised questionnaire to assess their awareness, knowledge, attitude and practices regarding breast feeding and their various practices. The data obtained from the survey was analyzed using various statistical tools such as frequency; percentage, mean and standard deviation were used.

Apart from these tools, independent $\mathrm{Z}$ Test, one way ANOVA were used for obtaining results. ANOVA was used to find out whether significant difference is there on the basis of means variables such as awareness, knowledge, attitude and practices scores across various categories formed on the basis of age, income, family size, education and $\mathrm{Z}$ test was used for finding whether the obtained means were different from the midpoint of various rating scales used in the questionnaire.

\section{Results and Discussion}

Surveys were conducted to assess the various aspects of mothers awareness, their knowledge, attitude, information regarding breastfeeding in different places, breast feeding practices among them, types of supplementary foods given to the child, prevalence of breast feeding practices and child diseases incidences respectively.

\section{Comparison of breastfeeding practices awareness, knowledge and attitude score across various categories}

Comparison of breastfeeding practices awareness score across various categories 
Comparison of breastfeeding practices knowledge score across various categories

Comparison of breastfeeding practices attitude score across various categories

\section{Comparison of breast feeding practices awareness, knowledge and attitude score across various categories}

For determining the awareness score among nursing mothers, they were asked to rate the 15 statements pertaining to breast feeding on the rating scale. The nursing mothers were asked to rate the statements from "Fully Aware" to "Not Aware" on the basis of their agreement. For analyzing the statements, a score of 5 was given to 'Fully Aware' and a score of 1 was given to 'Not Aware'. The comparative means scores of nursing mothers awareness regarding breast feeding were presented in the table 1.1 according to various parameters such as age, type of family and education.

It can be seen from the table 1.1 that the mean awareness score of all the nursing mothers is been categorized as follows where mean awareness score more than 55 is considered as high awareness score given ranking ' 3 ' followed by medium awareness score from 35 to 55 with ranking 2 and mean awareness score less than 35 is considered as low (ranking 1) respectively.

On the basis of age group, the nursing mothers were divided into four categories. It can be stated that the nursing mothers of the age group 20 to 25 years seemed to be quite aware about the breast-feeding practices. The mean awareness score of the nursing mothers, aged 20- 25 years came out to be highest i.e. 55.1 followed by the nursing mothers of aged between 25 to 30 years (mean score 53.9). The mean awareness score of nursing mothers of the age group between 30 to 35 years is found to be 53.7 and of the age group less than 20 years came out to be 50.1. It means that the above three age categories of the nursing mothers i.e. 25 to 30 years and 30 to 35 years and less than 20 years were having medium level of awareness regarding breastfeeding.

On the basis of educational qualification, the nursing mothers were divided into five categories. It can be seen from the table that the highest mean score of 67.1 was observed in case of postgraduates followed by the graduates (mean score- 61.5) and diploma holders (mean score 59.4). The mean awareness score of the nursing mother with matric and below is 51.4 and with $12^{\text {th }}$ standard is 49.4 fall in the medium level of awareness pertaining to breast feeding. There was a significant difference in the mean awareness score obtained by various categories segregated on the basis of the educational qualification $(p<0.01)$. It can be concluded from the results that mean awareness scores about breast feeding increased with increase in the educational level of the nursing mothers.

On the basis of type of family nursing mothers belonging to nuclear family were having highest mean score of 56.5 as compared to the nursing mothers that belong to the joint family(mean score- 53.1). There was a significant difference obtained between these two categories.

For determining the knowledge scores among nursing mothers, they were asked to rate the 15 statements pertaining to breast feeding on the rating scale. The nursing mothers were asked to rate the statements from "Strongly Agree" to "Strongly Disagree" on the basis of their agreement. For analyzing the statements, a score of 5 was given to 'Strongly Agree' and a score of 1 was given to 'Strongly Disagree'. The comparative mean knowledge scores of nursing mothers regarding breast feeding were 
presented in the table 1.2 according to various parameters such as age, type of family and education.

It can be seen from the table 1.2 that the mean knowledge score of all the nursing mothers is been categorized as follows where mean knowledge score more than 55 is considered as high knowledge score given ranking ' 3 ' followed by medium knowledge score from 35 to 55 with ranking 2 and mean knowledge score less than 35 is considered as low (ranking 1) respectively.

On the basis of age group, the nursing mothers were divided into four categories. It can be stated that the nursing mothers of the age group 30 to 35 years seems to be quite knowledgeable about the breast-feeding practices. The mean knowledge score of the nursing mothers, aged 30- 35 years came out to be highest i.e., 65.2 followed by the nursing mothers of aged between 25 to 30 years (mean score 63.8). The mean knowledge score of nursing mothers of the age group between 20 to 25 years is found to be 61.6 and of the age group less than 20 years came out to be 58.4. There was a significant difference in the mean knowledge score obtained by various categories segregated on the basis of the age groups $(\mathrm{p}<0.01)$. It can be observed that all the categories of the nursing mothers were very knowledgeable regarding breastfeeding.

On the basis of educational qualification, the nursing mothers were divided into five categories. It can be seen from the table that the highest mean knowledge score of 63.6 is observed in case of graduates followed by the $12^{\text {th }}$ standard (mean score 64.6) then postgraduates (mean score- 63.6), diploma holders (mean score 62.2) and matric and below (mean score 59.3) respectively. There is a significant difference in the mean awareness score obtained by various categories segregated on the basis of the educational qualification $(p<0.01)$. It can be concluded from the results that mean knowledge scores of nursing mothers regarding breast feeding does not depend on the increase in the level of education.

In contrast to this, the education of a mother seemed to directly have an impact on its child life. More educated mothers use more health services, provide better care for children, and have good knowledge of their child's proper feeding (Afrose et al., 2012). Simiarly to this another study revealed that It is evident that education is a key factor for mothers' knowledge of breastfeeding (Karimi et al., 2014).

On the basis of type of family nursing mothers belonging to joint family were having highest mean score of 63.3 as compared to the nursing mothers that belong to the nuclear family (mean score- 53.1). There was a significant difference found between these two categories showing high level of knowledge skills in both the categories. For determining the attitude score among nursing mothers, they were asked to rate the 15 statements pertaining to breast feeding practices on the rating scale. The nursing mothers were asked to rate the statements from "Strongly Agree" to "Strongly Disagree" on the basis of their agreement. For analyzing the statements, a score of 5 was given to 'Strongly Agree' and a score of 1 was given to 'Strongly Disagree'. The comparative mean attitude scores of nursing mothers regarding breast feeding practices were presented in the table 1.3 according to various parameters such as age, type of family and education. 
Table.1 Comparison of Breastfeeding Practices Awareness Score Across Various Categories $(\mathrm{N}=300)$

\begin{tabular}{|c|c|c|c|}
\hline \multicolumn{2}{|r|}{ Category } & \multirow{2}{*}{$\begin{array}{c}\text { Mean } \\
\text { Score } \pm \text { SD } \\
54.4 \pm 9.96\end{array}$} & \multirow{2}{*}{$\begin{array}{c}\text { F value/t-value } \\
\text { (p value) }\end{array}$} \\
\hline & Total $(\mathrm{N}=300)$ & & \\
\hline \multirow[t]{4}{*}{ Age } & $<20$ years $(\mathrm{n}=9)$ & $50.1^{\mathrm{a}} \pm 5.89$ & \multirow{4}{*}{$\begin{array}{c}0.95 \\
(0.41)\end{array}$} \\
\hline & $20-25$ years $(n=150)$ & $55.1^{\mathrm{a}} \pm 9.44$ & \\
\hline & $25-30$ years $(n=126)$ & $53.9^{a} \pm 10.96$ & \\
\hline & $30-35$ years $(n=15)$ & $53.7^{\mathrm{a}} \pm 8.96$ & \\
\hline \multirow[t]{5}{*}{ Education } & Matric and below $(n=111)$ & $51.4^{\mathrm{c}} \pm 6.96$ & \multirow{5}{*}{$\begin{array}{c}42.2 * * \\
(0.00)\end{array}$} \\
\hline & $10+2(\mathrm{n}=93)$ & $49.4^{c} \pm 10.03$ & \\
\hline & Graduate $(n=51)$ & $61.5^{b} \pm 7.28$ & \\
\hline & Post graduate and above $(n=28)$ & $67.1^{\mathrm{a}} \pm 4.31$ & \\
\hline & Diploma holder $(\mathrm{n}=17)$ & $59.4^{b} \pm 8.74$ & \\
\hline \multirow[t]{2}{*}{ Type of family } & Joint $(n=184)$ & $53.1^{b} \pm 10.93$ & \multirow{2}{*}{$\begin{array}{l}2.89 * * \\
(0.004)\end{array}$} \\
\hline & Nuclear $(n=116)$ & $56.5^{\mathrm{a}} \pm 7.83$ & \\
\hline
\end{tabular}

Table.2 Comparison of Breastfeeding Practices Knowledge Score Across Various Categories

\begin{tabular}{|c|c|c|c|}
\hline \multicolumn{2}{|c|}{ Category } & $\begin{array}{c}\text { Mean } \\
\text { Score } \pm \text { SD }\end{array}$ & $\begin{array}{c}\text { F value/t-value } \\
\text { (p value) }\end{array}$ \\
\hline & Total $(\mathbf{N}=\mathbf{3 0 0})$ & $\mathbf{6 2 . 6} \pm \mathbf{5 . 4}$ & \\
\hline Age & $<20$ years $(\mathrm{n}=9)$ & $58.4^{\mathrm{c}} \pm 8.96$ & $\begin{array}{c}7.36^{* *} \\
(0.00)\end{array}$ \\
\hline & $20-25$ years $(\mathrm{n}=150)$ & $61.6^{\mathrm{bc}} \pm 5.60$ & \\
\hline & $25-30$ years $(\mathrm{n}=126)$ & $63.8^{\mathrm{ab}} \pm 4.11$ & \\
\hline Education & $30-35$ years $(\mathrm{n}=15)$ & $65.2^{\mathrm{a}} \pm 7.36$ & \\
\hline & Matric and below $(\mathrm{n}=111)$ & $59.3^{\mathrm{c}} \pm 6.43$ & $23.64^{* *}$ \\
\hline & $10+2(\mathrm{n}=93)$ & $64.6^{\mathrm{a}} \pm 4.12$ & $(0.00)$ \\
\hline & Graduate $(\mathrm{n}=51)$ & $65.8^{\mathrm{a}} \pm 1.59$ & \\
\hline & Post graduate and above $(\mathrm{n}=28)$ & $63.6^{\mathrm{a}} \pm 2.83$ & \\
\hline Type of family & Diploma holder $(\mathrm{n}=17)$ & $62.2^{\mathrm{b}} \pm 3.52$ & \\
\hline & Joint $(\mathrm{n}=184)$ & $63.3^{\mathrm{a}} \pm 4.99$ & $2.71 * *$ \\
\hline & Nuclear $(\mathrm{n}=116)$ & $61.5^{\mathrm{b}} \pm 5.99$ & $(0.007)$ \\
\hline
\end{tabular}


Table.3 Comparison of Breastfeeding Practices Attitude Score Across Various Categories

$(\mathrm{N}=300)$

\begin{tabular}{|c|c|c|c|}
\hline \multicolumn{2}{|r|}{ Category } & $\begin{array}{l}\text { Mean Score } \\
+ \text { SD }\end{array}$ & \multirow{2}{*}{$\begin{array}{c}\text { F value/t-value } \\
\text { (p value) }\end{array}$} \\
\hline & Total $(\mathrm{N}=\mathbf{3 0 0})$ & $55.5 \pm 3.21$ & \\
\hline \multirow[t]{4}{*}{ Age } & $<20$ years $(\mathrm{n}=9)$ & $49.7^{\mathrm{C}} \pm 1.68$ & \multirow{4}{*}{$\begin{array}{l}22.9 * * \\
(0.00)\end{array}$} \\
\hline & 20- 25 years $(n=150)$ & $55.2^{\mathrm{a}} \pm 2.95$ & \\
\hline & $25-30$ years $(n=126)$ & $56.6^{\mathrm{a}} \pm 2.97$ & \\
\hline & $30-35$ years $(n=15)$ & $52.9^{\mathrm{b}} \pm 2.34$ & \\
\hline \multirow[t]{5}{*}{ Education } & Matric and below $(\mathrm{n}=111)$ & $54.3^{c} \pm 3.68$ & \multirow{5}{*}{$\begin{array}{l}10.1^{* *} \\
(0.00)\end{array}$} \\
\hline & $10+2(n=93)$ & $55.6^{\mathrm{b}} \pm 3.14$ & \\
\hline & Graduate $(\mathrm{n}=51)$ & $57.2^{\mathrm{a}} \pm 1.75$ & \\
\hline & Post graduate and above $(n=28)$ & $56.7^{\mathrm{ab}} \pm 2.00$ & \\
\hline & Diploma holder $(\mathrm{n}=17)$ & $55.9^{\mathrm{ab}} \pm 1.67$ & \\
\hline \multirow{2}{*}{ Type of family } & Joint $(\mathrm{n}=184)$ & $55.8^{\mathrm{a}} \pm 3.24$ & \multirow{2}{*}{$\begin{array}{l}2.12 * \\
(0.034)\end{array}$} \\
\hline & Nuclear $(n=116)$ & $55.0^{\mathrm{a}} \pm 3.12$ & \\
\hline
\end{tabular}

It can be seen from the table1.3 that the mean attitude score of all the nursing mothers is been categorized as follows where mean attitude score more than 58.7 is considered as strongly high score given ranking ' 1 ' followed by mean attitude score from 55.5 to 58.7 with ranking 2 . In addition to this mean attitude score less than 52.3 to 55.5 given rank 3 and ranking 4 is given when attitude mean score found to be 52.3 respectively.

On the basis of age group, the nursing mothers were divided into four categories. The mean attitude score of the nursing mothers, aged 2530 years came out to be highest i.e., 56.6 followed by the nursing mothers of aged between 20 to 25 years (mean score 55.2). The mean attitude score of nursing mothers of the age group between 30 to 35 years is found to be 52.9 and of the age group less than 20 years came out to be 49.7. There is a significant difference in the mean attitude score obtained by various categories segregated on the basis of the age groups $(p<0.01)$. It can be concluded that all the nursing mothers in their young adulthood i.e. between 25 to 35 years seems to have very good attitude except from the nursing mothers of the other categories. On the basis of educational qualification, the nursing mothers were divided into five categories. It can be seen from the table that the high mean attitude score of 57.2 is observed in case of graduates followed by the postgraduates (mean score- 56.7), diploma holders (mean score 55.9), $12^{\text {th }}$ standard (mean score 55.6), and metric and below (mean score 54.3) respectively. There is a significant difference in the mean attitude score obtained by various categories segregated on the basis of the educational qualification $(\mathrm{p}<0.01)$.

All the categories showing good attitude towards education qualification except one i.e. metric and below. Additionally, it is also observed from the results that mean attitude scores of nursing mothers regarding breast feeding does not depend on the increase in the level of education.

On the basis of type of family nursing mothers belonging to joint family were having high mean score of 55.8 as compared to the nursing mothers that belong to the nuclear family (mean score- 55). There is a significant 
difference found between these two categories.

\section{Acknowledgement}

This research paper is the output of Minor Research Project entitled' Breast Feeding: A Step towards Healthy and Productive Life' supported by Impress, ICSSR, New Delhi.

\section{References}

Alison Mc-Fadden et al., (2019) Counselling interventions to enable women to initiate and continue breastfeeding: a systematic review and meta-analysis. Inter Breastfeeding J 14(42):1-19.

Afrose, L., B. Banu, K. R. Ahmed and K. Khanom. (2012) Factors associated with knowledge about breastfeeding among female garment workers in Dhaka city. WHO South East Asia J. Public Health 1(3): 249.

American Academy of Pediatrics (2012) Policy Statement: breastfeeding and the use of human milk. Pediatrics129: e827-e41.

Craig H J (2010) Too scary to think about': first time mothers' perceptions of the usefulness of antenatal breastfeeding education. Journal of Australian College of Midwives 23(4):160-5.

Datta P (2009) Introduction to pediatric Nursing: Pediatric Nursing. Jayapee brotherspublication.2nd edition: pp1215

Joseph F, Ilop and Earland J (2019) A qualitative exploration of the sociocultural determinants of exclusive breastfeeding practices among rural mothers, North West Nigeria. International Breastfeeding Journal. $38: 1-7$.

Joseph Singh, et al., Breastfeeding practices among lactating mothers: Problems and prospects in a cross-sectional study. Int J Health Allied Sci 2012; 1:54-8

Karimi, B., M. Z. Sani, R. Ghorbani and N. Danai. (2014) The pregnant mothers' knowledge about breastfeeding in Semnan, Iran. Middle East J. Rehabil. Health 1(1): 20833.

Milinco, et al., (2019) Prevalence of breastfeeding in a baby-friendly pediatric practice: an experience in Trieste, Italy. International Breastfeeding Journal 26:239-34.

WHO, UNICEF (2018) The extension of the 2025 maternal, infant and young child nutrition targets to 2030. Geneva: World Health Organization.

World Health Organization (2009) Infant and young child feeding: model chapter for textbooks for medical students and allied health professionals. Geneva: World Health organisation

\section{How to cite this article:}

Vandana Gupta. 2021. Awareness, Knowledge and Attitude of Breast-Feeding Practices among Post Natal Mothers. Int.J.Curr.Microbiol.App.Sci. 10(08): 210-218. doi: https://doi.org/10.20546/ijcmas.2021.1008.025 